\title{
Stellar Photospheres and Molecules A View From The Bridge
}

\author{
David L. Lambert \\ Department of Astronomy, University of Texas, Austin, Texas 78712 USA
}

\section{Introduction}

The allegory alluded to in the subtitle of my review came to mind in the early morning hours when I was battling insomnia after a successful observing run at our McDonald Observatory. Similar bouts with insomnia are more widely classified as "jetlag," a condition probably too familiar to many of the readers!

Imagine two villages connected by a bridge spanning a deep, fast-flowing river. In my village on the south bank live the astronomers. Across the bridge live the chemical physicists and the physical chemists who are engaged in determining by all possible theoretical and experimental means the structure and spectra of molecules (and atoms). The astronomical community can be sorted into various clans of whom I consider here only the stellar spectroscopists and, in particular, the small subclan fascinated by quantitative spectroscopy who to pursue their trade must establish and maintain excellent communications with the village on the other side of the bridge. The necessity for contacts between the two villages has grown with the development of the tools of the stellar spectroscopists' trade: e.g., the advances of telescopes and spectrographs with respect to coverage of the electromagnetic spectrum and sensitivity, and the theoretical developments in the construction of model stellar atmospheres and the computation of synthetic stellar spectra. The recent decades of astronomical advances have been matched by exciting years in the village across the bridge: e.g., tunable lasers have spawned a renaissance in molecular (and atomic) spectroscopy, and theoretical work on molecular structure and spectra has advanced with the awesome growth of computers. Yet, a curious paradox may be sketched. These welcome and remarkable developments in the two villages have made it more difficult to maintain effective communications across the bridge. How can I possibly exploit fully the successes of MRD-CI calculations and appreciate their limitations? My counterparts across the bridge will echo my question: What experiments or quantum calculations might be usefully made in support of the astronomers' programs ATLAS, MARCS, SOSMARCS, and their relatives?

This review is one attempt to sketch the needs of stellar spectroscopists for basic molecular data. If we are to deserve collaborations with chemists and physi- 
cists, it is helpful if the astrophysical problems are of fundamental importance. The "stellar environment" presents a rich array of such problems. The stellar spectrum is our observational tool for understanding the physical structure and chemical composition of the stellar atmosphere from the deepest photospheric layers to the outermost limits of the stellar wind. The data primarily but not exclusively relating to the chemical composition are then applied to questions in stellar evolution and nucleosynthesis. Information gathered star-by-star is then used to tackle questions such as "How do stars evolve?" "How has the Galaxy evolved?" "How, when, and where were the chemical elements synthesized?" These are fundamental questions.

Here, I follow this introduction with a sketch of asymptotic giant branch (AGB) stars which are an especially interesting class of red giants whose environments can only be analysed when full attention is given to the molecular component of the stars' spectra. After explaining the structure and significance of these stars, I give examples of the basic molecular data needed in the analysis of stellar spectra, and I draw in large measure on personal examples. This review is directed primarily at the villagers across the bridge. In the last chapter, I return to my allegory and offer a few (obvious) remarks on how communication across the bridge might be made more effective.

\section{AGB Stars: A Key Site of Nucleosynthesis}

\subsection{Evidence of Nucleosynthesis}

Supernovae of Type II and Type Ia are widely recognized as major sites of nucleosynthesis. Type II SN are the terminal phase for massive stars $\left(M>8 M_{\odot}\right)$. Type Ia SN are commonly supposed to result when two white dwarfs coalesce to form an unstable compact object whose mass violates Chandrasekhar's limit for the maximum mass supportable by degenerate electrons. The AGB stars make major and distinctive contributions to stellar nucleosynthesis. In particular, these stars contribute elements that are not obviously synthesized by the SN: lithium, fluorine, and many heavy nuclides. AGB stars have initial masses in the range of about 1 to $8 M_{\odot}$; stars less massive than about $1 M_{\odot}$ have not yet exhausted their hydrogen supply, and stars more massive than $8 M_{\odot}$ evolve to die as $\mathrm{SN}$ and do not attain the structure of an AGB star.

The role of the AGB stars was demonstrated long before the structure of the stars was understood even in bare outline. Merrill (1952) provided this demonstration with his discovery of absorption lines of Tc $\mathrm{I}$ in blue spectra of a class of red giants known as $\mathrm{S}$ stars. Technetium was the signal of internal nucleosynthesis because this element has no stable isotopes. All isotopes are short-lived in the astronomical sense: e.g., ${ }^{99} \mathrm{Tc}$, the longest lived at low temperatures, has a half-life of about 200,000 yr., which is several orders of magnitude shorter than the time taken by the progenitors of typical $S$ stars to exhaust their supply of hydrogen and evolve to become AGB stars. Relative to normal (M-type) stars of similar temperature, spectra of $\mathrm{S}$ stars also show an enhancement of other 
heavy elements such as Zr and La; the latter enhancements are readily revealed on low dispersion spectra from strengths of $\mathrm{ZrO}, \mathrm{LaO}$, and other bands. Merrill's observational evidence for the synthesis of heavy nuclides was paired a few years later with the theoretical idea that synthesis occurs through successive captures of neutrons using abundant iron-group nuclei as the seeds for this synthesis. This is termed the s-process, where $s$ denotes "slow" which in turn implies that unstable neutron-rich nuclides will, in general, $\beta$-decay rather than experience another neutron capture. The operation of the $s$-process was described in the classic paper on nucleosynthesis by Burbidge et al. (1957). An excellent recent introduction is offered by Käppeler et al. (1989). Key unanswered questions about the s-process in AGB stars remain: What is the neutron source? What is the neutron density at the s-process site? Do models of AGB stars reproduce the observed pattern of $s$-process enrichments in individual AGB stars and in the mixture of stellar ejecta represented by unevolved stars of various ages and in the well-characterized solar system (carbonaceous chondrites)? What mass range among the $\mathrm{AGB}$ stars are the leading contributors of $s$-process nuclides to the Galaxy?

\subsection{The Structure of AGB Stars}

Stars do not become red giants until the initial $\mathbf{H}$ in their core is burnt to helium. Exhaustion of $\mathrm{H}$ terminates the main sequence phase of a star's life: main sequence lifetimes are $t_{\mathrm{MS}} \propto\left(M / M_{\odot}\right)^{-3}$, where the solar lifetime is about $10 \mathrm{Gyr}$. The remaining lifetime of a star is 10 percent or less of $t_{\mathrm{MS}}$. Unable to generate energy by nuclear fusion, the He core contracts and concomitantly the $\mathrm{H}$-rich outer layers expand and cool; the star becomes a red giant. The $\mathrm{He}$ core ignites eventually and $\mathrm{He}$ is burnt to $\mathrm{C}$ via the $3 \alpha$-process $\left(3^{4} \mathrm{He} \rightarrow{ }^{12} \mathrm{C}+\right.$ energy). The upper mass limit for AGB stars is set by the requirement that the $\mathrm{C}-\mathrm{O}$ core after $\mathrm{He}$ exhaustion be supported against gravitational collapse by the pressure of degenerate electrons. This mass limit is about $8 M_{\odot}$.

Stars more massive than $8 M_{\odot}$ ignite their C-O cores and proceed to a Type II SN. The lower mass limit for AGB stars of (presently) slightly less than $1 M_{\odot}$ is set by the age of the Galaxy ( $t_{\mathrm{gal}} \simeq 10-16 \mathrm{Gyr}$.) and the long main sequence lifetimes of low mass stars; obviously, $t_{\mathrm{MS}}(M) \simeq t_{\mathrm{gal}}$ sets the lower mass limit for AGB stars.

AGB stars are a study of sharp contrasts. The $\mathrm{C}-\mathrm{O}$ core of about $1 M_{\odot}$ has a radius approximately equal to that of the Earth; the mass density is about $10^{6}$ $\mathrm{g} \mathrm{cm}^{-3}$. The outer radius of the AGB star may exceed the radius of the Earth's orbit around the Sun. Even with one to several solar masses the mean density of the H-rich envelope is very low. The H-rich envelope is almost completely convective. This has important consequences for observers because, if mixing occurs between He-shell and the base of the envelope, convection will carry the products of nucleosynthesis into the spectroscopically accessible atmosphere. The Tc discovered by Merrill shows that such mixing can occur.

AGB stars must live by nuclear energy. Two potential sources exist: the thin He-shell around the degenerate $\mathrm{C}-\mathrm{O}$ core, and the $\mathrm{H}$-rich layers just exterior to 
the He-shell. As shown long ago by Schwarzschild \& Härm (1967), this doubleshell source is unstable in the sense that neither the $\mathrm{H}$-burning layers nor the $\mathrm{He}$ shell can burn uninterruptedly. Calculations show that the two nuclear sources burn approximately out of phase. For the majority of the time, $H$ burns at the base of the H-rich envelope. Helium, the ash of $\mathrm{H}$-burning, is added to the $\mathrm{He}$ shell. After an interval of $10^{4}-10^{5} \mathrm{yr}$, compression and heating of the He-shell causes it to ignite. On ignition, the He-shell is almost fully convective for a brief interval ( $\sim 10^{2}$ yr.). Following this "thermal instability" or "thermal pulse," $\mathrm{He}$ is burnt quiescently at the base of the thin (now radiative) shell. After a short period, He-burning can no longer be sustained, H-burning resumes, and the cycle of H-burning, the thermal instability, and He-burning is repeated. The cycle for intermediate mass AGB stars is illustrated in Fig. 1. The number of cycles experienced by an AGB star depends on several factors and is, in nature, likely to be severely determined by the mass loss rate, a factor ill-determined from observations and not yet predictable from theory. AGB evolution appears to terminate in a rapid and severe shedding of the $\mathrm{H}$-rich envelope known as the "superwind."

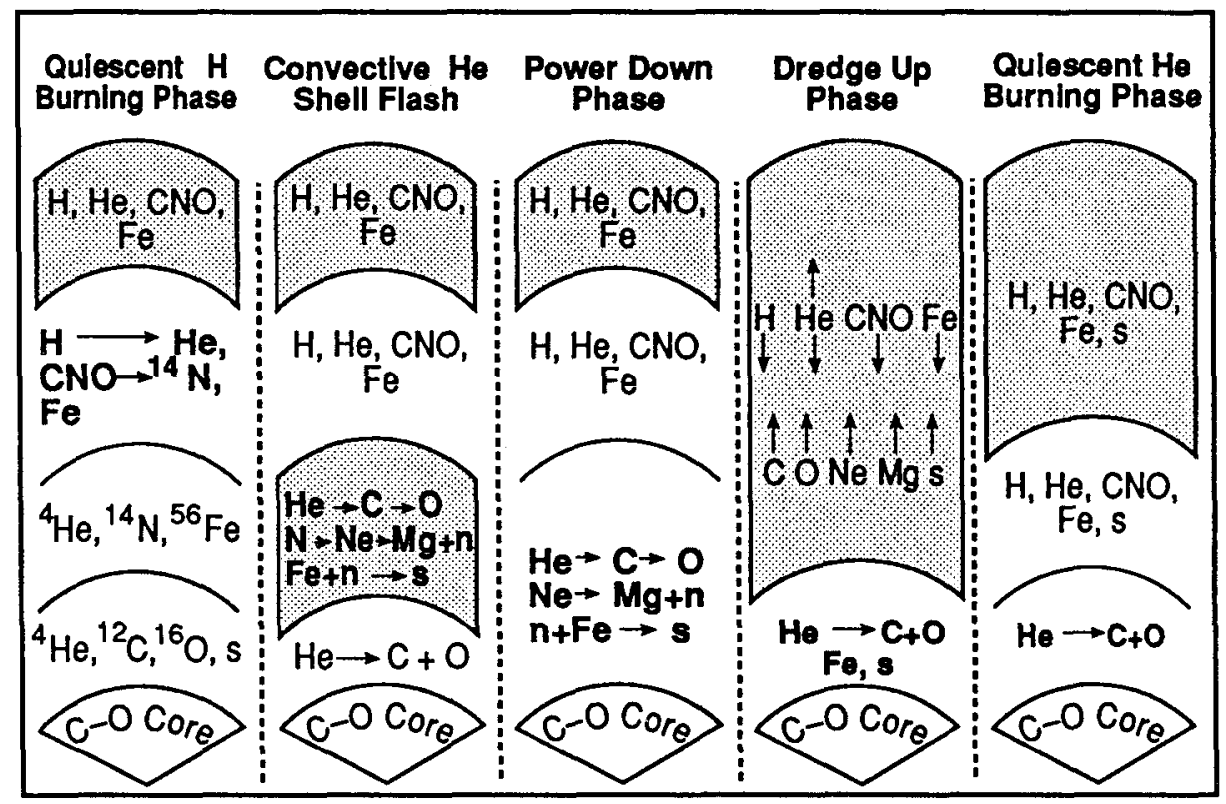

Fig. 1. Nucleosynthesis and dredge-up in an intermediate mass AGB star. This is a highly schematic representation that is not to scale in either the radial distance or mass coordinate, but instead emphasizes the ordering of dominant nuclear processes. The screened areas represent convective zones. The outer and $\mathrm{H}$-rich convective zone extends all the way to the surface. Regions of nuclear burning are shown in bold type. (After Iben 1985.) 


\subsection{The Third Dredge-Up}

The nuclear reactions comprising H-burning, He-burning, and accompanying reactions change the composition of the interior. These changes are observable only if the deep convective envelope can penetrate into the regions exposed to nuclear reactions. This penetration, if it occurs, leads to a phenomenon known as "the third dredge-up." Figure 2 depicts a successful dredge-up. (The first dredgeup occurs in all stars as they first become red giants or supergiants following the termination of the main sequence. The second dredge-up is predicted to occur in intermediate mass stars after exhausting of their He core as blue supergiants and on the return to the domain of the red giants). The $S$ and other red giants are incontrovertible evidence that the third dredge-up occurs in nature; the $s$ process, as I sketch below, accompanies He-burning. The theoretical evidence for the third dredge-up is mixed!



Fig. 2. Mixing regions as a function of time and activation of neutron sources during two thermal pulses in a low mass AGB model. Fully convective zones are denoted by screened areas. The times at which the ${ }^{13} \mathrm{C}$ and ${ }^{22} \mathrm{Ne}$ neutron sources are activated are indicated by the vertical lines within the convective shells (after Hollowell \& Iben 1989.)

An opportunity for the third dredge-up exists during the brief interval when He burns quiescently. At this time the base of the convective envelope will descend into the top of the He-shell. For the observer, the critical issue is the depth of penetration into the He-shell. Freshly synthesized ${ }^{12} \mathrm{C}$ with a little ${ }^{16} \mathrm{O}$ and 
the products of the s-process were distributed over the lower parts of the He-shell by the convection associated with the thermal pulse. If the convective envelope can penetrate into these parts, now quiescent and not convective, ${ }^{12} \mathrm{C}$ and $s$ process products will be added to the envelope and so to the atmosphere. A successful third dredge-up is sketched in Figure 2. Repeated applications of the third dredge-up increase steadily the $\mathrm{C}$-to-O ratio and the s-process enrichment of the atmosphere. The classical S stars such as those discovered by Merrill to contain $\mathrm{T} c$ have a higher $\mathrm{C} / \mathrm{O}$ ratio than less evolved red giants. When the $\mathrm{C} / \mathrm{O}$ ratio exceeds unity, C-containing molecules (e.g., $\mathrm{C}_{2}, \mathrm{CN}, \mathrm{HCN}$ ) dominate the spectrum of these "carbon" stars. Spectra of the S stars and their less evolved counterparts, the $\mathrm{M}$ stars, are dominated in the optical region by bands of oxides (e.g., $\mathrm{TiO}, \mathrm{ZrO}, \mathrm{LaO}$ ). The switch at $\mathrm{C} / \mathrm{O}=1$ from oxides to $\mathrm{C}$-containing molecules is engineered by the $\mathrm{CO}$ molecule. The He abundance of the envelope is increased even if the dredge-up does not penetrate the layers that experienced the thermal instability. In contrast to $\mathrm{C}$ and s-process elements, the $\mathrm{He}$ en richment of the envelope is quite minor. The reason for this is that $\mathrm{C}$ and s-process elements in the He-shell are overabundant by a factor of about 1,000 relative to their initial abundances in the envelope, but $\mathrm{He}$ is overabundant by the modest factor of 10 (by number of atoms). This is fortunate because the $\mathrm{He}$ abundance of the atmosphere is not directly measurable and, hence, it is reassuring to suppose the He abundance is not altered significantly by the third (or the preceding) dredge-up. Of course, the possibility exists that He-rich atmospheres may go undetected until almost all the $\mathrm{H}$ has been converted to $\mathrm{He}$ or removed!

A successful third dredge-up of ${ }^{12} \mathrm{C}$ and $s$-process elements was demonstrated theoretically for the more massive AGB stars by Iben (1975- see the comprehensive review papers Iben $[1985,1991])$. In some quarters, these stars of about 3 to 8 solar masses are called intermediate mass (IM) stars. The $s$ process is driven by the neutron source ${ }^{22} \mathrm{Ne}(\alpha, n){ }^{25} \mathrm{Mg}$ which is ignited in the He-shell at temperatures $T \gtrsim 3 \times 10^{8} \mathrm{~K}$. In the third dredge-up, the convective envelope skims off the top of the layers exposed to He-burning and the s-process. These products of nucleosynthesis are then mixed throughout the envelope including the surface. At the next thermal pulse, the remainder of the exposed layer in the He-shell is re-heated and re-exposed to neutrons; this re-exposure is characterized by an overlap factor often denoted by $r$. This sequence of re-exposures leads in a natural way after a few pulses to the dredged-up material being characterized by an exponential distribution of exposures to neutrons (Ulrich 1973). Such a distribution characterizes the solar/meteoritic abundances of $s$-process elements heavier than about Rb (the "main" s-process elements). In early calculations (Iben 1975) it was suggested that the ${ }^{22} \mathrm{Ne}$ neutron source and the predicted overlap factors led to a solar-like distribution of sprocess elements in the envelopes of these massive AGB stars. Furthermore, the numbers of these stars and the mass of their envelopes which are ejected before the stellar cores die as white dwarfs appeared in combination to suggest that these stars are the Galaxy's principal supplier of the "main" s-process elements (Truran \& Iben 1977). Unfortunately, this conclusion is now in doubt, in part because the competition to the ${ }^{22} \mathrm{Ne}$ 
neutron source from ${ }^{22} \mathrm{Ne}(\alpha, \gamma){ }^{26} \mathrm{Mg}$ was probably underestimated. This serves as a reminder that quantitative stellar spectroscopists in pursuit of the sites of stellar nucleosynthesis look across the river to nuclear physicists as well.

In the low mass $\left(M \lesssim 3 M_{\odot}\right)$ AGB stars, the temperatures in the He-shell are too low during the thermal pulses for the ${ }^{22} \mathrm{Ne}$ neutron source to be very effective. The principal source is most likely to be ${ }^{13} \mathrm{C}(\alpha, n)^{16} \mathrm{O}$. Questions remain as to how the necessary ${ }^{13} \mathrm{C}$ supply is generated; protons from the base of the convective envelope are mixed or diffused into the top of the He-shell during the long interpulse period of $\mathrm{H}$-burning. Then ${ }^{12} \mathrm{C}(p, \gamma)^{13} \mathrm{~N}\left(e^{-} \nu\right)^{13} \mathrm{C}$ provides the ${ }^{13} \mathrm{C}$ which is later ingested into the convective regions of the He-shell when He-burning resumes. Thanks to the paucity of protons, little ${ }^{13} \mathrm{C}$ is converted by proton capture to ${ }^{14} \mathrm{~N}$, as occurs in $\mathrm{H}$-burning when protons are abundant. At present, the ${ }^{13} \mathrm{C}$ abundance at He-burning is uncertain theoretically. As Figure 2 implies, the ${ }^{22} \mathrm{Ne}$ source may be ignited following the ${ }^{13} \mathrm{C}$ source in low mass AGB stars, but the neutron flux from the ${ }^{22} \mathrm{Ne}$ source is relatively weak. Note that a comparable uncertainty about the initial ${ }^{22} \mathrm{Ne}$ concentration in the $\mathrm{He}$ shell of an IM AGB star does not exist, because the ${ }^{22} \mathrm{Ne}$ abundance is set by the initial abundance of $\mathrm{C}, \mathrm{N}$, and $\mathrm{O}$ (and ${ }^{22} \mathrm{Ne}$, a minor species) which by $\mathrm{H}$-burning is converted to ${ }^{14} \mathrm{~N}$, and prior to He-burning ${ }^{14} \mathrm{~N}$ is converted by 2 $\alpha$-captures to ${ }^{22} \mathrm{Ne}$.

The reader is invited to read some recent papers to see how the issue of ${ }^{13} \mathrm{C}$ seeding of the He-shell is approached in theoretical calculations: e.g., Boothroyd \& Sackmann (1988), Hollowell \& Iben (1989), Busso et al. (1992). To the observer there would seem to be two potentially observable consequences of the degree of ${ }^{13} \mathrm{C}$ seeding. One, a high seeding means a high neutron exposure at each pulse and relatively higher production ratio of the heavier to the lighter s-process elements (say La to $\mathrm{Sr}$ ), as long as the overlap factor is unchanged. Second, the neutron density during s-processing is dependent on the rate at which ${ }^{13} \mathrm{C}$ is ingested into the He-burning convective layers. A high ${ }^{13} \mathrm{C}$ abundance tends to drive the $s$-process at a high neutron density. I discuss below the prospects for diagnosing the neutron density from analysis of the surface enrichments of key nuclides.

\subsection{Hot Bottom Burning in Very Luminous AGB Stars}

In the preceding look at nucleosynthesis in AGB stars, the focus was on the Heshell in which the ${ }^{12} \mathrm{C}$ and the $s$-process elements are made and whose presence in the atmosphere of an AGB star is often demonstrable from low resolution qualitative spectroscopy. H-burning at the base of the convective envelope also provides for nucleosynthesis. This contribution is potentially spectacular in two principal ways: an excess of $\mathrm{C}$ over $\mathrm{O}$ may be eliminated as ${ }^{12} \mathrm{C}$ is converted to ${ }^{14} \mathrm{~N}$ and a carbon star is reconverted to a now $\mathrm{N}$-rich $\mathrm{S}$ star, and a reservoir of ${ }^{3} \mathrm{He}$ is converted to ${ }^{7} \mathrm{Li}$ to create super $\mathrm{Li}$-rich stars that are quite possibly a major source of $\mathrm{Li}$ in the Galaxy.

$\mathrm{H}$-burning at the base of the convective envelope is a central feature of AGB evolution except in the short intervals when the He-shell is ignited. Although 
these episodes of H-burning convert some ${ }^{12} \mathrm{C}$ to ${ }^{14} \mathrm{~N}$ and consume ${ }^{3} \mathrm{He}$, no ${ }^{7} \mathrm{Li}$ is then or later injected into the convective envelope; the convective envelope does not penetrate the H-burning layer. The reason is very simple: the ${ }^{7} \mathrm{Li}$ is destroyed by protons as fast as it is produced. Also, the conversion of ${ }^{12} \mathrm{C}$ to ${ }^{14} \mathrm{~N}$ is constrained by the fact that the mass in the $\mathrm{H}$-burning layer is finite.

As an AGB star evolves to higher and higher luminosities, a structural change is predicted to occur. The $\mathrm{H}$-burning layer and the convective envelope join so that products of nucleosynthesis in the H-burning layer are swept to the surface and fresh "raw materials" are converted into the layer for nuclear processing. This episode is referred to by various phrases: I shall label it "hot bottom burning" or HBB - see Uus (1973) and Scalo et al. (1975) for an early description, and Blöcker \& Schönberner (1991) and Sackmann \& Boothroyd (1992) for more recent calculations of evolution and/or accompanying nucleosynthesis.

The possibility that ${ }^{7} \mathrm{Li}$ may be synthesized in red giants was recognised by Cameron \& Fowler (1971), who proposed the "7Be-transport mechanism" in which ${ }^{7} \mathrm{Li}$ is created via the reaction chain ${ }^{3} \mathrm{He}(\alpha, \gamma){ }^{7} \mathrm{Be}\left(e^{-}, \nu\right)^{7} \mathrm{Li}$, where the ${ }^{3} \mathrm{He}$ is a product of $\mathrm{H}$-burning in the main sequence phase and the ${ }^{7} \mathrm{Li}\left(\right.$ and ${ }^{7} \mathrm{Be}$ ) is transported by convection to cooler layers to avoid destruction by protons; ${ }^{7} \mathrm{Li}$ destruction occurs very rapidly at the temperatures necessary to induce conversion of ${ }^{3} \mathrm{He}$ to ${ }^{7} \mathrm{Be}$. The ${ }^{3} \mathrm{He}$ was created by the pp-chain of $\mathrm{H}$-burning. ${ }^{3} \mathrm{He}$ production occurs very slowly because the initial reaction $p\left(p, e^{+} \nu\right)^{2} \mathrm{H}$ proceeds via the weak interaction; the nuclear reaction ${ }^{2} \mathrm{H}(p, \gamma)^{3} \mathrm{He}$ is much faster. ${ }^{3} \mathrm{He}$ is produced in significant quantities only in main sequence stars. Calculations show that, as the star evolves to become a red giant, the ${ }^{3} \mathrm{He}$ produced avoids destruction over a thick zone outside the $\mathrm{H}$-burning core and later the $\mathrm{H}$-burning shell. It is this reservoir of ${ }^{3} \mathrm{He}$ that is available to the HBB AGB star for processing to ${ }^{7} \mathrm{Li}$. Since the reservoir is finite, the rise of the surface ${ }^{7} \mathrm{Li}$ abundance of an $\mathrm{HBB}$ AGB star may be followed by a decline as the ${ }^{7} \mathrm{Li}$ is destroyed on its return to the H-burning layer at the base of the convective envelope and replenishment is impossible owing to exhaustion of the ${ }^{3} \mathrm{He}$ reservoir. I comment below on $\mathrm{Li}$-rich AGB stars and the role of molecular spectra in the analysis of atomic ( $\mathrm{Li} \mathrm{I}$ ) lines.

\section{Structure and Spectra of Molecules and Stars}

\subsection{Why Study Molecules in Stellar Photospheres?}

To the easily intimidated, the association of atoms into molecules results in a spectrum of awful complexity. This complexity must be faced by the observer curious about AGB stars. Indeed, molecular spectra offer novel diagnostics of AGB behaviour not provided by the atomic lines. In this section, I shall note briefly the unique contributions of molecular spectra to the analysis of photospheric structure and composition and then turn to discussion of the data on molecular structure and data needed to effect the analyses of AGB stars. (I shall not discuss the chromospheres and circumstellar shells of AGB stars, see reviews by Olofsson, Hinkle, and by Sedlmayr, this volume, on that subject). Not unexpectedly, my emphasis is on the roles of molecules in determining photospheric 
chemical compositions in order to address questions concerning stellar evolution and nucleosynthesis. Applications of molecules to the observational definition of the physical properties of stellar photospheres are important but are not covered in detail. Molecular spectra provide information on the chemical composition and the physical structure of the photosphere. These two aspects are coupled, as is well shown by Jørgensen (this volume). I shall not discuss this coupling except to remark that the molecular line blanketing, which is dependent on chemical composition, is a principal determinant of the (theoretical) structure of an AGB star's photosphere. Specification of the blanketing calls for much of the molecular data needed in the analysis of photospheric spectra.

Molecular spectra may be used to estimate the temperature and pressure of the photosphere. In practice, their use as barometers is rare. Here, one seeks to use molecules in an analogous way to the use of atomic and ionic spectra (say, Fe I and Fe II lines) with the Saha ionization equilibrium to derive the stellar surface gravity; for example, one might use the infrared $\mathrm{OH}$ rotation-vibration lines with the forbidden [O I] 6300 and $6363 \AA$ lines and dissociation equilibrium to derive the surface gravity of an AGB star (the assumption of hydrostatic equilibrium links the gas pressure affecting the dissociation equilibrium to the surface gravity). In practice, this application of molecular spectra is very difficult for AGB stars because the necessary available atomic lines are few in number and often severely blended. Applications are possible for warmer red giants where molecular lines are less numerous. In AGB stars, one might envision applications of diatomic with triatomic molecules as barometers, say $\mathrm{OH}$ and $\mathrm{H}_{2} \mathrm{O}$ in O-rich stars or $\mathrm{CN}$ and HCN in C-rich stars.

Spectroscopists have long used the measured equivalent widths of lines of an atom (or ion) to derive an excitation temperature $\left(T_{\text {exc }}\right)$ for that species. If LTE is valid, this $T_{\text {exc }}$ is obviously a weighted mean of the kinetic temperature over the layers contributing to the lines' formation. In recent years, comparison of the measured $T_{\text {exc }}$ and predictions from model atmospheres has been used to derive a star's effective temperature $\left(T_{\text {eff }}\right)$. Space prohibits a thorough critique of the atomic lines as a stellar thermometer. While valuable and valid estimates of $T_{\text {eff }}$ are provided for many types of stars, there are often severe limitations to this application of atomic lines. A majority of the limitations are rooted in fundamentals of atomic structure and spectra.

Use of atomic or molecular lines as a thermometer providing $T_{\text {exc }}$ and, thence, $T_{\text {eff }}$ requires that there be available an adequate collection of weak lines sampling a range in excitation potential, possessing accurately known $g f$-values, and drawn from levels whose populations show no significant departures from LTE. The latter constraint is cited because, with few exceptions, predictions of non-LTE populations for atoms in stellar atmospheres have not advanced beyond a level that I call "semi-quantitative" and do not permit the $T_{\text {exc }}$ (NLTE)- $T_{\text {eff relation }}$ to be predicted. This is especially true for cool stars and those atomic species frequently adopted as thermometers (e.g., Fe I, Ti I). My list of qualitative conditions could be translated to quantitative conditions embracing the number of lines, the range in excitation potential, the accuracy of the $g f$-values, and the 
precision of the stellar equivalent widths.

My intent is not to explore the quantitative accuracy of $T_{\text {exc }}$ estimates, but to point out that molecules offer inherent advantages over atoms. Molecules are more practical thermometers than atoms. In particular, the rotational structure of a given band of an electronic or rotation-vibration transition provides a set of lines whose relative $f$-values may often be fixed to satisfactory accuracy from a rotational line strength (Hönl-London factor) and a quantity describing the rotation-vibration interaction. The range in excitation potentials, which controls the sensitivity of the thermometer, may be extended by combining bands provided that their relative $f$-values are known. Preliminary theoretical studies indicate that the rate constants for rotational and vibrational excitation and de-excitation through $\mathrm{H}, \mathrm{He}, \mathrm{H}_{2}$, and e collisions dominate the statistical equilibrium of the ground and, possibly, the excited vibrational states of a molecule resident in the photosphere of a red giant (Thompson 1973; Hinkle \& Lambert 1975); i.e., the local excitation and kinetic temperatures are identical or almost so.

By contrast, atomic spectra are far less attractive thermometers. An atomic line list spanning an adequate range in excitation potential will necessarily involve different terms which are most probably connected by strong permitted radiative transitions and, hence, potentially subject to non-LTE effects. A satisfactory line list may also span a broader wavelength interval than the molecular alternative. In general, the available supply of accurate atomic $f$-values will be found wanting. Then, in the case of cool stars, the supply of atomic lines will be depleted because several to many potential lines will be masked by strong molecular absorption (the high density of molecular lines will also mean that a molecular thermometer may be compromised by the paucity of unblended weak molecular lines).

As a final note on molecules and the atmospheric structure, I mention the use of molecules as probes of the photospheric velocity field - see, for example, the analyses by Tsuji (1988) of the CO $2.3 \mu \mathrm{m}$ rotation-vibration lines in the spectra of $M$ giants showing the radial velocity to vary systematically with a line's excitation energy. These shifts and line asymmetries show that "a pack of low excitation $\mathrm{CO}$ lines should be originating in an extra molecular component, not yet expanding at large velocity, but well distinct from the photosphere" (Tsuji 1988). As Tsuji points out, this zone revealed by the CO lines may be the environment in which dust formation occurs and mass loss is initiated by radiation pressure on the grains.

To date, the contributions of observed molecular lines to our understanding of the chemical composition exceed the insights obtained into the physical structure of AGB stars. I divide these prelusive remarks into two parts: isotopic ratios and elemental abundance ratios.

Three concise statements suffice to show why molecular lines in stellar spectra are analysed for the isotopic abundance ratio of one or more of the molecule's constituent atoms:

1. Often, the isotopic ratios address astrophysical questions of great import, 
e.g., stellar nucleosynthesis of the chemical elements.

2. Often, ratios of high accuracy are obtainable with limited knowledge of the atmospheric structure and a partial description of the basic molecular data, e.g., the ${ }^{12} \mathrm{C} /{ }^{13} \mathrm{C}$ ratio may be extracted from $\mathrm{CN}$ lines without a precise value for the molecule's dissociation energy whose persistent uncertainty afflicts all attempts to use the CN lines as monitors of either the $\mathrm{C}$ or the $\mathrm{N}$ elemental abundances.

3. In general, the detectable molecules in stellar spectra provide isotopic ratios for those atoms for which the isotopic atomic wavelength shifts are too small to be resolved in stellar spectra. This fortunate circumstance arises because the nuclear masses have a direct influence on a molecule's rotation-vibration energy levels.

A combination of isotopic with elemental abundance ratios is often essential in addressing the key astrophysical questions. In quantitative analyses of warm/solar stars, molecular spectra may supplement the data on elemental abundance ratios provided by atomic spectra. In the cooler stars such as the inhabitants of the AGB, however, molecular spectra may be the primary or even the sole source of elemental abundance ratios. Certainly, for the CNO trio whose abundances reflect the presence of $\mathrm{H}$ - and He-burning products in the stellar atmosphere, molecules are the key to the abundances. Note too that a simultaneous analysis of two or more indicators may be required for a single elemental abundance. Consider, for example, the C-rich AGB stars. Carbon monoxide and $\mathrm{N}_{2}$ largely control the partial pressures of free $\mathrm{C}, \mathrm{N}$, and $\mathrm{O}$ and, hence, of molecules containing these elements. While the $\mathrm{CO}$ lines are a direct measure of the $\mathrm{O}$ abundance $\epsilon(\mathrm{O})$, the $\mathrm{C}_{2}$ lines reflect the difference $(\epsilon(\mathrm{C})-\epsilon(\mathrm{O}))^{2}$ and, hence, the $\mathrm{C}$ abundance is set by the combination of $\mathrm{CO}$ and $\mathrm{C}_{2}$ lines. An obvious extension of this to $\mathrm{CN}$ shows that the $\mathrm{N}$ abundance requires a simultaneous fit to $\mathrm{CO}, \mathrm{C}_{2}$, and $\mathrm{CN}$ lines. A corollary is obvious: errors in the basic data ( $f$-values and dissociation energies) for a molecule may affect the abundance of more than one element, and the effects may not be equal. For example, an error in the $f$-value of the $\mathrm{C}_{2}$ lines contributes the following errors:

$$
\begin{gathered}
\Delta \log (\epsilon(\mathrm{C})-\epsilon(\mathrm{O})) \sim 0.5 \Delta \log f\left(\mathrm{C}_{2}\right) \\
\Delta \log \epsilon(\mathrm{N}) \sim \Delta \log f\left(\mathrm{C}_{2}\right)
\end{gathered}
$$

where the CN lines are the source of the $\mathrm{N}$ abundance and "all" of the $\mathrm{N}$ is supposed to be associated into $\mathrm{N}_{2}$ (i.e., $p\left(\mathrm{~N}_{2}\right) \propto \epsilon(\mathrm{N})$ and $p(\mathrm{~N})^{2} \propto p\left(\mathrm{~N}_{2}\right)$, hence $\epsilon(\mathrm{N}) \propto p(\mathrm{CN})^{2}$ where $p$ denotes a partial pressure $)$.

The spectrum of an AGB star may provide novel information for the molecular spectroscopist because the star's photosphere is a spectacular light source whose composition, column density, and combination of low pressure and moderate temperatures cannot be realized in the laboratory. As evidence, I cite our discovery of $\mathrm{ZrS}$ bands in 7,000-10,000 $\AA$ spectra of $\mathrm{S}$ stars (Lambert \& Clegg 1980) ahead of laboratory detections of these bands. The necessity for laboratory work is well shown by the recent work on TiS using a King-type furnace (Jonsson 
et al. 1992). The TiS bands from 1.05 to $1.35 \mu \mathrm{m}$ were shown to account quite well for bands previously attributed (Hinkle et al. 1989) to $\mathrm{ZrS}$ on the basis of wavelengths predicted by $a b$ initio calculations (Langhoff \& Bauschlicher 1988). Analysis of high resolution stellar spectra will now be needed to show if the stellar band are a mix of 'TiS and $\mathrm{ZrS}$ lines. Before this can be done, the predicted infrared bands of $\mathrm{ZrS}$ will have to be detected in the laboratory.

\subsection{Molecules as Irritants and Stimulants}

Civil servants in the villages on either side of the bridge would assuredly suppose that the interests of the astronomers in one village and of the physicists and chemists across the river in the other village would be efficiently served if the astronomers compiled a list of their needs for basic molecular data, and if the physicists and chemists prepared a catalogue of available data with a supplement describing products that could be made on special request. Then, messengers would be detailed to meet at the mid-point of the bridge and to exchange the list and the catalogue. In my experience such an impersonal exchange is rarely fruitful. Therefore, I decline to offer a list of current needs for basic molecular data. I elect to present a few examples from our recent pursuit of AGB stars where molecular spectra either interfered with the primary atomic line in the investigation ("molecules as irritants") or the molecular lines were themselves the key to a fundamental question of stellar evolution and nucleosynthesis ("molecules as stimulants"). It will be clear that readily available molecular data are often inadequate for the purposes of the stellar analyses.

Lithium Synthesis by HBB AGB Stars. Lithium production by very luminous AGB stars with an H-burning occurring at the base of the convective envelope may be a major contributor to the lithium budget of the Galaxy. Our recent observations of AGB stars in the Small and Large Magellanic Clouds showed, as predicted, that Li-production is confined to the most luminous AGB stars (Smith \& Lambert 1989, 1990; Smith et al. 1993). This result could not be demonstrated from the well-known and brighter examples of super Li-rich AGB stars in our Galaxy because their distances and, hence, their luminosities are illdetermined. For the Magellanic Clouds, our nearest neighbours among galaxies, many independent and generally concordant methods have been applied to the measurement of their distances. As the distances of the AGB stars in the Clouds are known, reliable luminosities may be estimated. In order to assess the role of these AGB stars as lithium producers, we need to estimate the $\mathrm{Li}$ abundance of their photospheres from analysis of the Li i lines (molecules containing Li-e.g., LiH - are unlikely to be present in detectable quantities).

Inspection of Fig. 3 shows the obvious presence of a bandhead of the $\mathrm{TiO}$ $\gamma(1-0) \mathrm{R}_{1}$ band. The Li I $6707 \AA$ resonance doublet is in a region crossed by $\mathrm{TiO}$ lines from, for example, the $\mathrm{R}_{2}$ and $\mathrm{R}_{3}$ branches of the $\gamma(1-0)$ band. This interference from $\mathrm{TiO}$ lines must be taken into account. $\mathrm{TiO}$ lines are of such a high density in this spectral region that lines overlap to simulate a quasicontinuous opacity that depresses the apparent continuum level. A few stronger 
TiO lines may also be blended with the Li I $6707 \AA$ feature, and they too must be taken into account. Today, the standard tool for such an abundance analysis is the synthetic spectrum in which a comprehensive list of atomic and molecular lines is combined with a model photosphere and the predicted spectrum matched to the observed spectrum.

An example of a fit of a synthetic spectrum to an observed spectrum is shown in Figure 4. The reader may find the details of this analysis in Plez et al. (1993). Here, I wish to draw attention to the depression of the local continuum: the relative flux near the $\mathrm{Li}$ i feature is approximately 0.4 , where the continuum flux in the absence of lines is at 1.0. The $60 \%$ depression of the continuum is due to $\mathrm{TiO}$ lines which populate this region at a line density of several hundred lines per $\AA$.

Many of these lines have not been observed in laboratory spectra, but their wavelengths are predicted from molecular constants and their oscillator strengths estimated from a few available radiative lifetimes (see Schamps this volume). Indeed, except through selective laser excitation the individual lines will not be observable in laboratory spectra; they will overlap to form quasi-continuous emission or absorption just as their stellar counterparts do. This example shows clearly that stellar demands may outstrip the present capabilities of laboratory spectroscopy. And $\mathrm{TiO}$ is a molecule extensively investigated in the laboratory!

Correct representation of the $\mathrm{TiO}$ blanket is acute for lithium because in many red giants, the $6707 \AA$ feature is the sole evidence for lithium. In the case of iron and other abundant elements with rich atomic spectra, one may select the spectral region so that molecular line blanketing is minimized. Happily, the $\mathrm{Li}$ abundance of these Li-rich stars is sufficiently high that a subordinate line at $8126 \AA$ is seen in a region of weak blanketing; the local continuum is depressed by only 5 to $10 \%$ and not $60 \%$ as at $6700 \AA$. Since the $8126 \AA \mathrm{Li}$ I line is weak, it is important to identify completely the few contributing $\mathrm{TiO}$ (and other) blending lines. The available laboratory spectroscopy appears adequate for this purpose (Fig. 5).

Rubidium and Neutron Densities in Thermally Pulsing He Shells. As a second example of "molecules as irritants" I cite the case of the Rb I $7800 \AA$ resonance line and the use of the Rb abundance to measure the neutron density in the thermally pulsing He-shell that drives the s-process. Rubidium shares with its fellow alkali lithium the characteristic that it is of low abundance and the neutral atom has a low ionization potential. Such characteristics mean that generally only the strongest resonance lines are potentially detectable. For $\mathrm{Rb}$ I these lines are at 7800 and $7947 \AA$. In spectra of O-rich AGB stars, the $7947 \AA$ line is irretrievably blended. By thorough accounting of the molecular lines (mostly $\mathrm{TiO}$ ) and a blending $\mathrm{Si}_{\mathrm{I}}$ line it is possible to extract the $\mathrm{Rb}$ abundances from the $7800 \AA$ line. Our early work on $\mathrm{Rb}$ was done on Barium giants (Tomkin \& Lambert 1983; Smith \& Lambert 1984; Malaney \& Lambert 1988) which are considerably warmer than the AGB stars. Analyses of Barium giants are relevant to studies of AGB stars because these giants are created when 


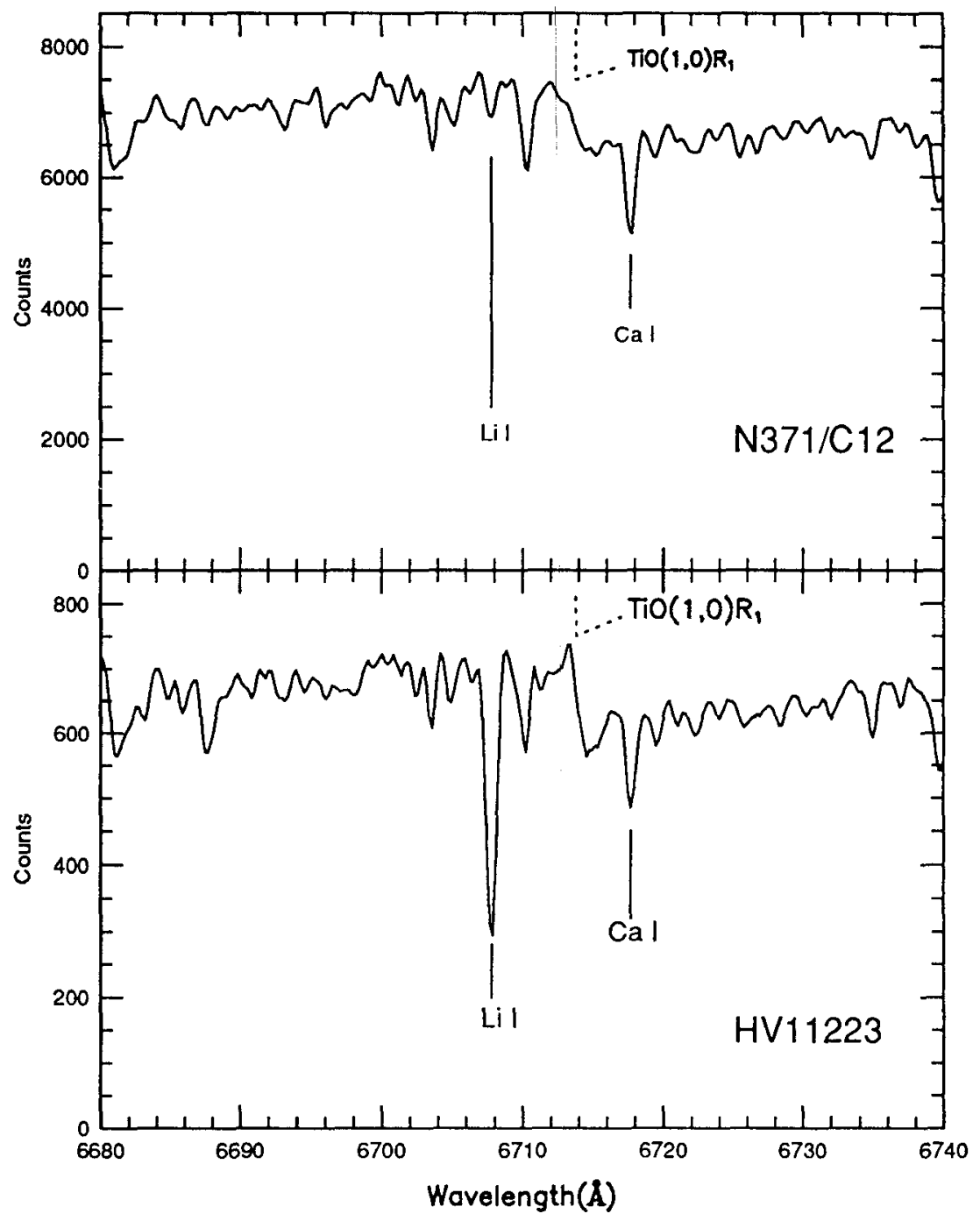

Fig. 3. Spectra of an AGB star HV11223 and a supergiant N371/C12 in the small Magellanic Cloud. N371/C12 is a cool massive star with a temperature and surface gravity similar to that of the AGB star. Note the general similarity of the spectra; the TiO (1-0) $R_{1}$ band and the $\mathrm{Ca}$ I line have quite similar strength in the two stars. The one striking difference is the strength of the Li I $6707 \AA$ feature in the AGB star. 




Fig. 4. Observed (dots) and calculated (line) spectrum of the AGB star HV1963. Two TiO 1-0 R bandheads are clearly seen. Note the continuum in the absence of lines is at a relative flux of 1.0 .

an AGB star transfers large amounts of mass to a companion to convert it to the Barium star. The C-O core of the AGB star remains as a white dwarf in orbit around the Barium star. Since the Barium giant and its main sequence predecessor have warm photospheres, molecular contamination of the spectra is greatly reduced permitting analyses that are impossible for AGB stars. Recently, we have completed analyses of $\mathrm{Rb}$ in a sample of O-rich AGB stars.

Why is $\mathrm{Rb}$ of especial interest? To answer this question we must examine the path taken by the s-process along the valley of nuclear stability. In the limit that the neutron density $\mathrm{N}(\mathrm{n})$ driving the $s$-process tends to zero, the s-process takes a unique path; I ignore here the possibility that the temperature and electron density may influence the path. The uniqueness results from the fact that the first $\mathbf{n}$-rich unstable nuclide of an element decays rather than captures an additional neutron. At the neutron densities expected in the thermally pulsing He-shell, however, an additional neutron capture may occur before an unstable nuclide decays. This competition between neutron capture and (usually) $\beta$-decay creates a potential branchpoint in the $s$-process path. For each branchpoint, there is a critical value of the neutron density $N(n)_{c}$ : if $N(n) \ll N(n)_{c}$, the unstable nuclide at the branchpoint decays, but if $\mathrm{N}(\mathrm{n}) \gg \mathrm{N}(\mathrm{n})_{c}$, the nuclide experiences a neutron capture. This difference between the low and high neutron density 


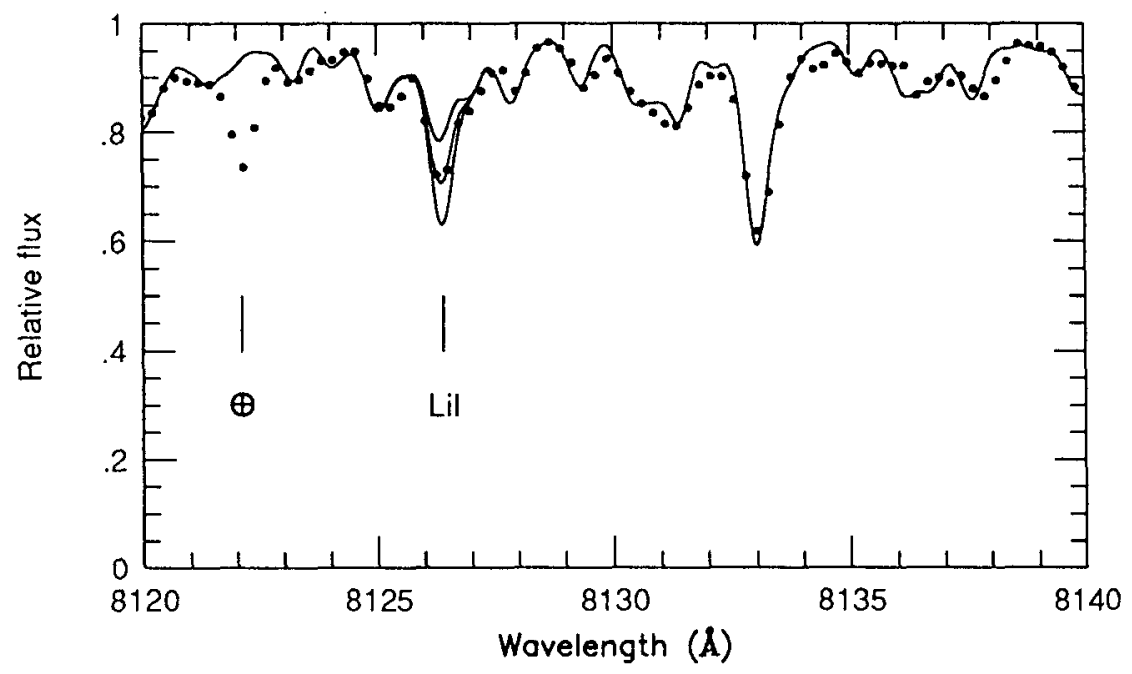

Fig. 5. The observed (dots) and synthetic spectrum (line) of HV1963 near the Li I $8126 \AA$ line. Synthetic spectra are shown for three Li abundances: the best fit and $\pm 0.5 \mathrm{dex}$ from that fit. The observed feature at $8122 \AA$ is due to terrestrial water vapor.

paths affects the yields from the s-process of nuclides immediately following the branchpoint. Then, there is the prospect of measuring the photospheric abundances of the affected nuclides and, hence, inferring the neutron density that directed the $s$-process. Just two branches are available for use with AGB stars:

1. the branch at unstable ${ }^{85} \mathrm{Kr}$ that determines the $\mathrm{Rb}$ abundance relative to its neighbours $\mathrm{Sr}$ and $\mathrm{Y}$.

2. the branch at unstable ${ }^{95} \mathrm{Zr}$ that controls synthesis of the stable isotope ${ }^{96} \mathrm{Zr}$.

Here, I comment further on ${ }^{85} \mathrm{Kr}$. The interested reader may find discussion of ${ }^{95} \mathrm{Zr}$ and of the detection of isotopic bandheads of $\mathrm{ZrO}$ in S stars in Smith (1988) and Lambert (1988).

Operation of the ${ }^{85} \mathrm{Kr}$ branchpoint, which is illustrated in Fig. 6. , is complicated slightly by the fact that ${ }^{85} \mathrm{Kr}$ has a short-lived state which reduces the effectiveness of the branch run by ${ }^{85} \mathrm{Kr}$ with a long-lived ground state. A benefit resulting from the complication is that the influence of ${ }^{85} \mathrm{Kr}$ on the s-process path is sensitive to the duration of the neutron burst in the thermal pulse. The potential branch at ${ }^{86} \mathrm{Rb}$ plays a minor role. Beer \& Macklin (1989) provide accurate measurements of the ${ }^{85} \mathrm{Rb}$ and ${ }^{87} \mathrm{Rb}$ neutron capture cross-sections and discuss operation of the ${ }^{85} \mathrm{Kr}$ branchpoint in AGB models.

Exploitation of the branchpoint for AGB stars must rely on its effect on the $\mathrm{Rb}$ abundance. In the limit $\mathrm{N}(\mathrm{n}) \rightarrow 0$, the $s$-process path is through ${ }^{85} \mathrm{Rb}$, ${ }^{86} \mathrm{Rb}$ to ${ }^{86} \mathrm{Sr}$. In the high density limit, the path runs through ${ }^{86} \mathrm{Kr}$ to ${ }^{87} \mathrm{Rb}$ and ${ }^{88} \mathrm{Sr}$. Thus, the elemental abundances of $\mathrm{Rb}$ relative to $\mathrm{Sr}$ or $\mathrm{Y}$ is sensitive to 
neutron densities around $\mathrm{N}(\mathrm{n})_{c}$. Thanks to the fact that the neutron capture cross-sections of ${ }^{85} \mathrm{Rb}$ and ${ }^{87} \mathrm{Rb}$ (see Fig. 6) differ by a factor of about 20 , the $\mathrm{Rb} / \mathrm{S} r$ and $\mathrm{Rb} / \mathrm{Y}$ ratios vary by more than an order of magnitude between the low and high $N(n)$ limits.

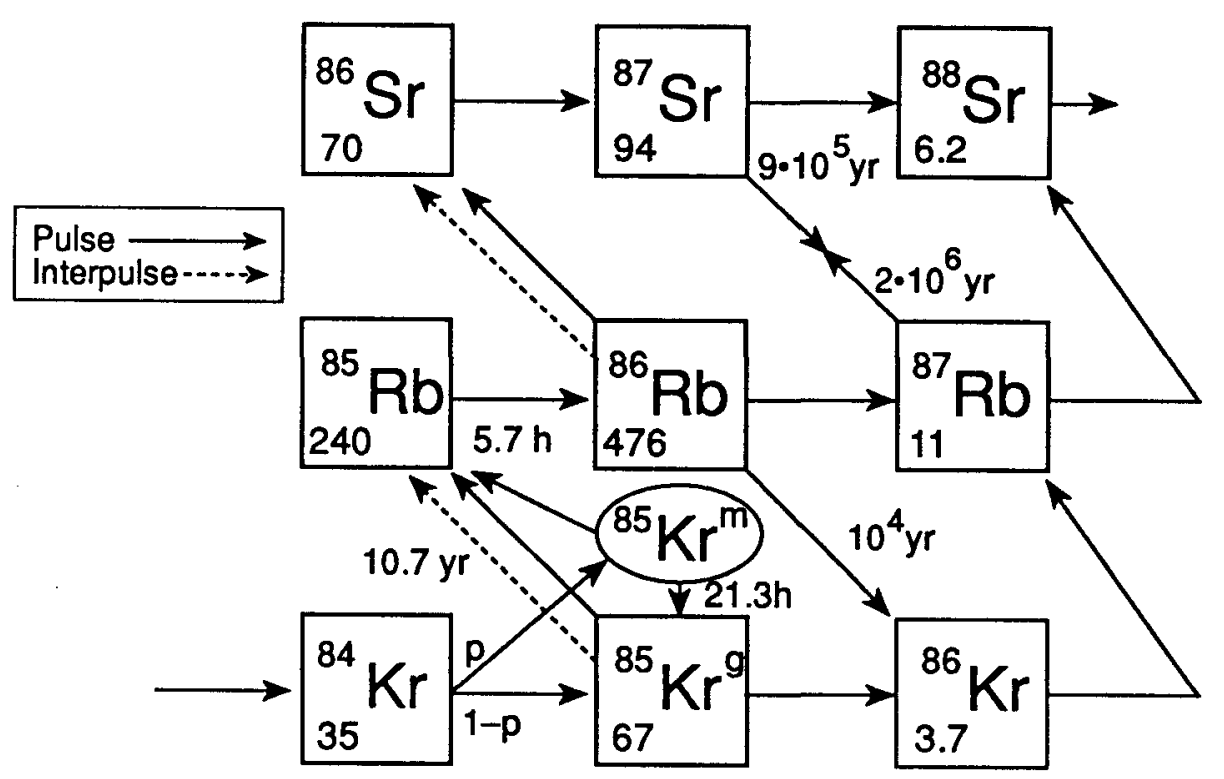

Fig. 6. Synthesis of $\mathrm{Kr}, \mathrm{Rb}$, and $\mathrm{Sr}$ isotopes during a thermal pulse (solid line) and in the interpulse phase (dashed lines) when the ${ }^{85} \mathrm{Kr}$ and ${ }^{86} \mathrm{Rb}$ which are built up decay. The population $P$ of the isomeric state in ${ }^{85} \mathrm{Kr}$ is generated by neutron capture on ${ }^{84} \mathrm{Kr}$ $(P=0.49 \pm 0.06)$. The time scales for ${ }^{87} \mathrm{Rb}$ and ${ }^{87} \mathrm{Sr} \beta$-decay during the pulse are too long to be of any significance for the synthesis. The branching of ${ }^{86} \mathrm{Rb}$ to ${ }^{86} \mathrm{Kr}$ can be neglected too. (After Beer \& Macklin 1989.)

Our analyses of $\mathrm{Rb}$ in Barium giants and directly in O-rich AGB stars (Lambert \& Smith 1993) have shown no case indicative of a high N(n). Beer \& Macklin (1989) predicted $\mathrm{Rb} / \mathrm{Sr}$ ratios for the neutron pulses expected for intermediate mass AGB stars where ${ }^{22} \mathrm{Ne}(\alpha, n)^{25} \mathrm{Mg}$ is the neutron source. These pulses have a high $\mathrm{N}(\mathrm{n})\left(\gtrsim 3 \times 10^{9} \mathrm{~cm}^{-3}\right)$ and a rapid termination of s-process and a $\mathrm{Rb} / \mathrm{Sr}$ ratio is predicted in excess of that observed. Beer and Macklin show that pulses with a peak $\mathrm{N}(\mathrm{n}) \sim 10^{8}$ to $10^{9} \mathrm{~cm}^{-3}$ and pulse lengths $\Delta t \simeq 10 \mathrm{yr}$. are required to fit the observations. Pulses of this kind have been engineered for low mass AGB models where ${ }^{13} \mathrm{C}(\alpha, n)^{16} \mathrm{O}$ is the leading neutron source. We identify the stars examined by us for $\mathrm{Rb}$ as low mass AGB stars and are continuing to search for intermediate mass AGB stars rich in $\mathrm{Rb}$ (principally ${ }^{87} \mathrm{Rb}$ ) and ${ }^{96} \mathrm{Zr}$. 
MgH Bands and Mg Isotopic Ratios. In the chase for the $\mathrm{Li}$ and $\mathrm{Rb}$ abundances of AGB stars, the $\mathrm{TiO}$ molecule is an "irritant" (ZrO serves as a stimulant when it is used to derive the abundance of ${ }^{96} \mathrm{Zr}$ ). Here, I comment on the role the $\mathrm{MgH} A^{2} \Pi-X^{2} \sum^{+}$green system plays in the identification of the neutron source driving the $s$-process in the He-shell's thermal pulse. To the observer, $\mathrm{MgH}$ in this example is a "stimulant."

Two neutron sources are likely candidates: ${ }^{22} \mathrm{Ne}(\alpha, n)^{25} \mathrm{Mg}$ for $T \simeq 3 \times 10^{8} \mathrm{~K}$ and ${ }^{13} \mathrm{C}(\alpha, n){ }^{16} \mathrm{O}$ for $T \simeq 1.5 \times 10^{8} \mathrm{~K}$. The higher ignition temperature for ${ }^{22} \mathrm{Ne}(\alpha, n)$ means that it runs the s-process in intermediate mass AGB stars. ${ }^{13} \mathrm{C}(\alpha, n)$ is the primary source of neutrons in low mass AGB stars. The ${ }^{22} \mathrm{Ne}$ source cannot easily run the s-process and elude the stellar spectroscopist because it leads to a non-solar mix of the $\mathrm{Mg}$ isotopes in the He-shell and, after dredge-up of s-process products, in the photosphere of an IM-AGB (Truran \& Iben 1977; Scalo 1978; Malaney 1987); in a typical case, the photospheric ratios of ${ }^{24} \mathrm{Mg}:{ }^{25} \mathrm{Mg}:{ }^{26} \mathrm{Mg}$ may be 1:4:4 for a factor of 10 enrichment of s-products at the surface instead of the star's initial ratios (presumed close to solar) of 8:1:1. The $\mathrm{MgH}$ lines provide an opportunity to measure the isotopic ratio. Operation of the ${ }^{13} \mathrm{C}$ neutron source makes no significant alteration to the $\mathrm{Mg}$ isotopic ratio, and the additional amount of ${ }^{16} \mathrm{O}$ synthesized in the $\mathrm{He}$-shell is negligible relative to that from He-burning through ${ }^{12} \mathrm{C}(\alpha, \gamma){ }^{16} \mathrm{O}$.

Examination of $\mathrm{MgH}$ lines in about 15 Barium stars and several MS and S stars (see Lambert 1991 for references) shows none with the ${ }^{25} \mathrm{Mg}$ and ${ }^{26} \mathrm{Mg}$ enrichments expected from the ${ }^{22} \mathrm{Ne}$ source. The conclusion is that the examined stars are low mass AGB stars. It will be of interest to search for $\mathrm{MgH}$ lines in spectra of the Magellanic Cloud's Li-rich AGB stars whose masses according to pulsational calculations are high enough for the ${ }^{22} \mathrm{Ne}$ source to run the $s$-process. Perhaps the test should be applied to the immediate progenitors of these stars (i.e., AGB star not yet experiencing HBB), because there is a possibility that excess ${ }^{25} \mathrm{Mg}$ and ${ }^{26} \mathrm{Mg}$ in the convective envelope are removed by protons: ${ }^{25} \mathrm{Mg}(p, \gamma){ }^{26} \mathrm{Al}$ and ${ }^{26} \mathrm{Mg}(p, \gamma){ }^{27} \mathrm{Al}$. It appears, however, that significant Li-production (and preservation) occurs only for temperature in the hot bottom too cool to destroy ${ }^{25} \mathrm{Mg}$ and ${ }^{26} \mathrm{Mg}$. Conversely, if ${ }^{25} \mathrm{Mg}$ and ${ }^{26} \mathrm{Mg}$ are destroyed in very hot bottomed envelopes, $\mathrm{Li}$ is not added to the envelope and the atmosphere in detectable amounts.

C, N, O Abundances and Infrared Spectroscopy. Stellar spectroscopists were long confined to the spectral regions detectable with photographic plates. Exploration of nucleosynthesis occurring in AGB stars was severely compromised by the lack of access to infrared spectra. One may cite three principal reasons for exploring the infrared (here, 1.0 to $5 \mu \mathrm{m}$ ):

- The AGB stars are cool and, hence, emit most of their flux in the infrared.

- The line density in infrared spectra may be lower than in visual spectra.

- The infrared is essential to the determination of the $\mathrm{C}, \mathrm{N}$, and $\mathrm{O}$ elemental and isotopic abundances that monitor the mixing of $\mathrm{H}$ - and He-burning products into the atmosphere. 
The third point deserves amplification in the message carried across the bridge from the astronomical village to the other side. To highlight the need for infrared spectroscopy, I shall compare the indicators of the $\mathrm{C}, \mathrm{N}$, and $\mathrm{O}$ abundances available for the $\mathrm{G}-\mathrm{K}$ giants and the AGB stars.

At the photospheric temperatures of $\mathrm{G}-\mathrm{K}$ stars, molecules mark but do not deface the visual spectrum. $\mathrm{C}, \mathrm{N}$, and $\mathrm{O}$ abundances are obtainable from visualto-near infrared $(\lambda \lesssim 9000 \AA)$ spectra. Lines that have been used include the $\mathrm{C}_{2}$ Swan system's $\Delta v=0$ and -1 bands, the CN Red system $\Delta v=2$ to 4 bands, and the [O I] 6300 and $6363 \AA$ lines (see for example, Lambert \& Ries 1981; Kjærgaard et al. 1982). Infrared lines could (and should) also be used: the CO rotation-vibration (or rovibrational for short) $\Delta v=2$ and 3 lines, the CN Red system's $\Delta v=0$ and -1 bands, and the $\mathrm{OH}$ rovibrational $\Delta v=1$ and 2 bands.

In the case of the O-rich AGB stars, the $\mathrm{C}, \mathrm{N}$, and $\mathrm{O}$ indicators in the visual are lost in a wealth of molecular lines (the CN Red system is often detectable among the other lines). Fortunately, the infrared remains a source of $\mathrm{C}, \mathrm{N}$, and $\mathrm{O}$ indicators. In addition to the list cited above for $\mathrm{G}-\mathrm{K}$ giants I would add the $\mathrm{NH}$ rovibrational lines. In the coolest stars, one should add the $\mathrm{H}_{2} \mathrm{O}$ rovibrational lines.

For C-rich AGB stars, the infrared is similarly of paramount importance. The principal indicators are the CO rovibrational $\Delta v=2$ and 3 lines, the $\mathrm{CN}$ Red system $\Delta v=-2$ and -3 lines, the $\mathrm{C}_{2}$ Phillips $\Delta v=-2$ and Ballik-Ramsay $\Delta v=0$ lines, the $\mathrm{CH}$ and $\mathrm{NH}$ rovibrational $\Delta v=1$ lines and, in the cooler stars, the rovibrational lines of $\mathrm{HCN}$ and $\mathrm{C}_{2} \mathrm{H}_{2}$.

Since the atmosphere of an AGB star is so extensive, the stellar spectra provide a more complete representation of these various transitions than has been obtained in the laboratory. In addition, the absolute $g f$-values of the transitions are needed. Much new data have been supplied in recent years, principally from calculations. But, as the discussion of CN (Costes \& Naulin this volume) see below too-indicates, some basic astrophysical needs remain unfulfilled. Laboratory infrared quantitative spectroscopy and quantum chemistry calculations of infrared transitions on astrophysically relevant molecules are to be encouraged.

\section{What Does the Village Store Need?}

This review, written to intrigue the villagers in the physical-chemical community across the river, began with a description of the contributions of AGB stars to nucleosynthesis and then turned to portraits of selected problems in which stellar spectroscopists have drawn on molecular spectra in pursuit of answers. In these pursuits, the incompleteness of the available data on the molecules of interest may become apparent and, in some cases, may severely limit the precision of the answers provided to the astrophysical questions. In the final section, I comment on two topics that deserve continuing attention by the physical-chemical community. The astrophysical village store needs to be supplied with the "products" that I describe now. 


\subsection{The Dissociation Energy of CN}

A red giant's photospheric $\mathrm{N}$ abundance is a tracer of the photosphere's contamination with the product's of H-burning. Recall that the CNO-cycle H-burning reactions turn $\mathrm{H}$ to $\mathrm{He}$ as they convert "all" initial $\mathrm{C}, \mathrm{N}$, and $\mathrm{O}$ nuclei into ${ }^{14} \mathrm{~N}$ nuclei, i.e., $\mathrm{H}$-burning creates $\mathrm{N}$-rich material. Evidence of this material at the surface of red giants is predicted and observed following the first and second dredge-ups. A HBB AGB star necessarily achieves further $\mathrm{N}$ enrichment. A full characterization of the $\mathrm{N}$ enrichment demands accurate measurement of the photospheric $\mathrm{N}$ abundance.

For all red giants except the very coolest, the $\mathrm{CN}$ molecule is effectively the sole indicator of the $\mathrm{N}$ abundance (of course, the $\mathrm{N}$ abundance cannot be obtained from $\mathrm{CN}$ alone but from combinations of $\mathrm{CN}$ with other C-containing molecules and even $\mathrm{O}$ indicators, as the $\mathrm{C}$ and $\mathrm{O}$ abundances are linked through CO molecule formation). The $\mathrm{NH} A-X 0-0$ band near $3360 \AA$ is unattractive as an $\mathrm{N}$ indicator because the red giants emit little flux in the ultraviolet and the spectrum is very crowded with lines. In the coolest red giants, the NH rovibrational lines near $3 \mu \mathrm{m}$ may be exploited. In short, knowledge of a giant's $\mathrm{N}$ abundance depends heavily on the reliability of the basic data for CN. One item required is the molecule's dissociation energy $D_{0}(\mathrm{CN})$. Our village store needs a "new improved" product, as I shall now sketch.

I consider the extreme case of the cool carbon stars where nitrogen atoms are almost fully associated in $\mathrm{N}_{2}$ molecules, and $\mathrm{O}$ is similarly associated into $\mathrm{CO}$ molecules. Then, the number density of CN molecules is related to the elemental $\mathrm{C}, \mathrm{N}$, and $\mathrm{O}$ abundances (here denoted by $\epsilon$ ) as follows:

$$
n(\mathrm{CN}) \propto\left(\epsilon_{c}-\epsilon_{o}\right) \epsilon_{N}^{\frac{1}{2}}
$$

where $\left(\epsilon_{c}-\epsilon_{o}\right)$ has to be determined from $\mathrm{C}_{2}$ lines. This relation shows that $\log \epsilon_{N} \propto 2 \log n(\mathrm{CN})$. The dissociation energy enters into the breakdown of $\mathrm{n}(\mathrm{CN})$ between the $\mathrm{N}$ abundance and the temperature:

$$
n(\mathrm{CN}) \propto 10^{D_{0}^{0} \theta}
$$

where $\theta=5040 / T$ and the temperature dependent partition functions are ignored. For cool carbon stars $\theta \simeq 2$. Then, an uncertainty $\Delta D_{0}^{0}$ (in $\mathrm{eV}$ ) contributes an abundance error $\Delta \log \epsilon_{N} \simeq 4 \Delta D_{0}^{0}$ where, for example, $\Delta D_{0}^{0} \simeq 0.25$ $\mathrm{eV}$ results in $\Delta \log \epsilon_{N} \simeq \pm 1$.

Since the total conversion of solar $\mathrm{C}, \mathrm{N}$, and $\mathrm{O}$ to $\mathrm{N}$ changes the $\mathrm{N}$ abundance by about $1.2 \mathrm{dex}$, it is clear that an uncertainty of \pm 1 dex severely limits our ability to detect even large additions of H-burnt material to a red giant's photosphere. The problem is alleviated for warmer red giants such as the $\mathrm{K}$ giants, which have experienced the first dredge-up because the $\mathrm{N}_{2}$ molecules are not a major sink of $\mathrm{N}$ and the temperatures are warmer. Nonetheless, the need for an accurate value of $D_{0}$ remains acute. The need can be met to some extent by the stellar spectroscopists' skill in devising differential methods of analysis whose results may be nearly independent of the adopted value of $D_{0}(\mathrm{CN})$. 
To answer the question "What is the "best" value of $D_{0}(\mathrm{CN})$ ?", a search of the literature would be made by the astrophysical villager. Fortunately, Huang et al. (1992) have listed published estimates of the heat of formation of $\mathrm{CN}$ $\left(\Delta \mathrm{H}_{f}(\mathrm{CN})\right)$ obtained from 1962 to 1990 from a wide variety of experiments including analysis of solar $\mathrm{CN}$ absorption lines and $a b$ inito quantum calculations. Note that $D_{0} \mathrm{CN}=\frac{1}{2} D_{0}^{0}\left(\mathrm{~N}_{2}\right)+\mathrm{L}(\mathrm{C})-\Delta \mathrm{H}_{f}(\mathrm{CN})$, where $\mathrm{L}(\mathrm{C})$ is the heat of sublimation of graphite and $D_{0}\left(\mathrm{~N}_{2}\right)=9.760 \pm 0.005 \mathrm{eV}$ and $\mathrm{L}(\mathrm{C})=7.353 \pm 0.02 \mathrm{eV}$ are established values (see JANAF Tables, Chase et al. 1984) Inspection of Huang et al.'s list shows the experimental values range from $D_{0}(\mathrm{CN})=7.66 \pm 0.05 \mathrm{eV}$ to $7.90 \pm 0.08 \mathrm{eV}$. Although the range is less than my example of $\pm 0.25 \mathrm{eV}$, it nonetheless is greater than the errors assigned to these (and most other entries) in the list.

Happily, the more recent entries do appear to be converging. Huang et al. (1992) estimate $D_{0}(\mathrm{CN})=7.72 \pm 0.03 \mathrm{eV}$ from photodissociation of $\mathrm{C}_{2} \mathrm{~N}_{2}$. Costes et al. (1990-see also Costes \& Naulin this volume) obtained $D_{0}(\mathrm{CN})=7.77 \pm$ $0.04 \mathrm{eV}$ from analysis of the energy of the $\mathrm{CN}$ molecules produced from $\mathrm{C}+\mathrm{NO}$ reactions.

The temptation to adopt $D_{0}(\mathrm{CN})=7.75 \pm 0.04 \mathrm{eV}$ is probably strong. However, not only do some experiments suggest a lower value but $a b$ initio calculations empirically corrected from calculations on similar molecules with well determined dissociation energies also suggest lower values: $D_{0}(\mathrm{CN})=7.53 \mathrm{eV}$ (Larsson et al. 1983, as quoted by Huang et al. 1992) and $D_{0}=7.65 \pm 0.06 \mathrm{eV}$ (Bauschlicher et al. 1988).

Recalling the case of the cool carbon stars $\left(\Delta \log \epsilon_{\mathrm{N}} \approx 4 \Delta D_{0}^{0}(\mathrm{eV})\right)$, one would like to get $\Delta D_{0}^{0} \lesssim 0.05 \mathrm{eV}$ so that the $\mathrm{N}$ abundance uncertainty from $D_{0}$ (CN) alone is less than $50 \%$. The suggestion that the recent experiments have reached this precision is not supported by refined $a b$ initio calculations. Are the experiments correct? Are the $a b$ initio calculations less reliable than estimated? Can a spectroscopic method be devised to provide-finally - an accurate value of $D_{0}(\mathrm{CN})$ ?

Analyses of stellar CN lines use the red $(A-X)$ system to the almost total exclusion of the violet $(B-X)$ system. These analyses require both an estimate of $D_{0}(\mathrm{CN})$ and the $f$-values of the appropriate red system bands. As for $D_{0}(\mathrm{CN})$, estimates of the $f$-values have shown a dismayingly large scatter. Larsson (this volume) reviews the status of measurements and $a b$ initio predictions of the radiative lifetimes of the vibrational levels of the $A^{2} \Pi$ state. The "best" experimental lifetimes $\left(\tau_{v^{\prime}}\right)$ including a laser-induced fluorescence experiment (Lu et al. 1992) give $\tau_{v}$-values that are consistently shorter than the most recent $a b$ initio predictions (e.g., Bauschlicher et al. 1988): the difference amounts to about $30 \%$ at $v^{\prime}=0$ but a factor of 3 for $v^{\prime}=7$. This discrepancy too deserves continued scrutiny by experimentalists and theoreticians. The variation of $\tau_{v}$, with $v^{\prime}$ or equivalently the run of band $f$-values across the red system may be well determined from analysis of the solar CN absorption lines Sauval et al. (1994). 


\subsection{Excitation by $\mathrm{H}$ Atoms?}

The assumption of Local Thermodynamic Equilibrium (LTE) so simplifies the analysis of stellar spectra and the construction of model stellar atmospheres that there is an understandable reluctance to abandon it in favor of the less restrictive assumption of Steady State Statistical Equilibrium or non-LTE. Under LTE, all local properties (excluding the radiation field) assume the values attained in thermal equilibrium at the local kinetic temperature. The power of LTE is that it reduces greatly the atomic and molecular data needed to construct model stellar atmospheres and to employ those atmospheres in the analysis of stellar spectra. In particular, LTE restricts the kind of data needed to lists of classified lines, their $g f$-values, and the photoionization (photodetachment) cross-sections for bound-free (and free-free) transitions contributing to the continuous opacity.

Departures from LTE must exist. Then, the LTE assumption that level populations may be calculated from the Boltzmann-Saha equation is dropped, and level populations are obtained from a series of rate equations that in turn are coupled to equations of transfer. If $\mathrm{N}(i)$ is the population of state $i$, the rate equation for $\mathrm{N}(i)$ is

$$
\frac{d N(i)}{d t}=0=-\sum_{i} N(i)\left(R_{i j}+C_{i j}\right)+\sum_{j} N(j)\left(R_{j i}+C_{j i}\right) .
$$

The level is considered to be depopulated by radiative processes (rate constants $R_{i j}$ ) and collisional processes (rate constants $C_{i j}$ ). The level $i$ is repopulated by radiative and collisional processes from other levels $j$. The set of levels includes various stages of ionization; for example, a non-LTE study of Fe I should consider radiative and collisional coupling of $\mathrm{Fe} \mathrm{I}$ and $\mathrm{Fe}$ II levels and, hence, an adequate treatment will require a model $\mathrm{Fe}$ atom and a $\mathrm{Fe}^{+}$ion. In cool stars, certain atoms and molecules have to be treated together.

It is obvious that introduction of non-LTE calls for a greater range of atomic and molecular data. In particular, collisional excitation, ionization, and dissociation cross-sections are needed. Calculation of the radiation fields that enter into the rates $R_{i j}$ will also call for more atomic and molecular data than was necessary in LTE; for example, the ultraviolet radiation field is unimportant to the calculation of a model atmosphere of a cool star because little flux is carried by the ultraviolet, but the ionization or excitation of an atom in non-LTE may be significantly affected by radiative rates involving the ultraviolet.

In cool stars, hydrogen atoms outnumber electrons by a wide margin with the ratio being roughly the abundance of the easily ionized metals. For the upper layers of the solar photosphere, $n(\mathrm{H}) / n(\mathrm{e}) \sim 10^{4}$ where electrons are contributed by $\mathrm{Mg}, \mathrm{Si}$, and $\mathrm{Fe}$ principally. Of course, the ratio $n(\mathrm{H}) / n(\mathrm{e})$ is even larger in metal-poor stars. One expects that all particles-electrons and $\mathrm{H}$ atoms--are describable by a single kinetic temperature. If, then, the fraction of particles with energies in excess of an excitation energy $E_{\mathrm{UL}}=E_{\mathrm{U}}^{A}-E_{\mathrm{L}}^{A}$ is the same for electrons and hydrogen atoms, why is it that excitation of $A$ is generally assumed to occur through e on $A$ collisions and not through collisions between $A$ and the 
far more numerous $\mathrm{H}$ atoms? It will strike the observant novice as curious that neglect of the $\mathrm{H}$ on $A$ collisions is almost never justified.

The rate constant for collisional excitation $A_{\mathrm{L}} \rightarrow A_{\mathrm{U}}$ is an integral of a velocity dependent cross-section $\sigma(v)$ over the Maxwellian velocity distribution; I write this rate constant as $\langle\sigma(v) v\rangle$. Certainly, the $\mathbf{H}$ atoms' contribution relative to that of the electrons is reduced by the ratio of the thermal velocities, i.e., $\left(m_{e} / m_{\mathrm{H}}\right)^{\frac{1}{2}} \simeq 1 / 43$. There is also a general argument to suggest that the crosssection for excitation by $\mathrm{H}$ atoms is likely to be less than that for excitation by electrons.

The general argument was given by Massey (1949) and was discussed recently by Anderson (1981). A collision occurs with a characteristic time scale $t \sim r / v$, where $r$ is the effective range and $v$ is the relative velocity. The transition in the perturbed atom corresponds to a frequency $v \sim \Delta E / h$. Classically and, also, quantum mechanically, the expectation is that the cross-sections for excitation and de-excitation will be small unless $1 / t \sim v$ or $t v \sim 1$. It is instructive to examine this limit:

$$
\begin{aligned}
t v=\frac{r}{v} \frac{\Delta E}{h} & =\frac{4 r \Delta E}{\sqrt{T}} \text { (electrons) } \\
& =\frac{170 r \Delta E}{\sqrt{T}}(\text { hydrogen atoms }) .
\end{aligned}
$$

In these formulae, the effective range is given in $\AA$ units and the energy $\Delta E$ in $\mathrm{eV}$. In the limit $t v \rightarrow 0$, the cross-section will decrease from a maximum near $t v \lesssim 1$, but it will remain significantly large for small $t v$ values. On the other hand, the cross-section is expected to be very small for $t v \gg 1$. A typical optical transition has $\Delta E \sim 3 \mathrm{eV}$ so that with $T \sim 5000 \mathrm{~K}$ and $r \sim 1 \AA, \mathrm{tv} \sim 1 / 6$ for an electron collision and $\sim 7$ for a hydrogen atom collision. Then, the cross-section for an inelastic collision with an $\mathrm{H}$ atom is expected to be much smaller than for an inelastic collision with an electron. The effective range of the $\mathbf{H}$-atom collision is possibly less than that of the e-atom collision. In an atom, fine-structure and some term-to-term transitions correspond to small $\Delta E$. In a molecule, the rotational and vibrational transitions correspond to small excitation energies: $\Delta E_{\text {rot }} \sim 0-0.05 \mathrm{eV}$ and $\Delta E_{\mathrm{vib}} \sim 0.2-0.4 \mathrm{eV}$ for typical molecules. When $\Delta E$ is much less than $1 \mathrm{eV}$, the product $t v<1$ and excitation by hydrogen (also helium) atoms must be considered.

The total rate appearing in the equations of statistical equilibrium is the product of $\langle\sigma v\rangle$ and the density of projectiles: can the small $\langle\sigma v\rangle$ expected for optical transitions $(\Delta E \gtrsim 1 \mathrm{eV})$ from collisions with $\mathrm{H}$ atoms be offset by the large ratio $n(\mathrm{H}) / n(\mathrm{e})$ ?

In a series of non-LTE calculations on Li I, Fe I, and other atoms, Holweger and colleagues have included the inelastic collisions with $\mathrm{H}$ atoms where the rate constants were taken from a generalization of a "modified classical Thomson formula" (Drawin 1968, 1969), which was expected to yield "an order of magnitude estimate of collisional excitation and ionization cross-sections." The Thomson formula alluded to is that for ionization of atoms by electrons. The reader interested in the employed rate constants and the empirical evidence from analysis 
of absorption lines in stars for the efficacy of collisions with $\mathrm{H}$ atoms is referred to Lambert (1993).

In view of the fact that the rate constants for the $\mathrm{H}$-atom collisions are based on a rudimentary estimate adapted from Thomson's (1912) classical estimate for collisional ionization by electrons, it is surely of interest to test the rudimentary estimate against experiments and quantum mechanical predictions. Such a test seems especially worthwhile when it is recalled that Thomson's estimate predicts cross-sections for collisional ionization by electrons that are considerably larger than the real cross-sections near threshold (Seaton 1962). What follows is a brief discussion of a theoretical estimate for excitation of the Li I $2 s^{2} S \rightarrow 2 p^{2} P$ resonance $(6707 \AA)$ transition and experimental measurements for excitation of the $(\mathrm{Na} D)$ transition.

Theoretical estimates of excitation through $\mathrm{H}$-atom collisions must consider the $\mathrm{H}$-atom pair as forming a temporary molecule and, hence, a set of molecular potentials. The $\mathrm{LiH}$ molecule is a relatively simple molecule as far as contemporary quantum chemistry is concerned. As a stellar spectroscopist with no more than a superficial knowledge of quantum chemistry, I would suppose that excitation rates by $\mathrm{H}$-atom collisions can now be estimated with fair precision. Allen \& Dickinson (private communication) have estimated the rate constants for excitation of the Li I $2 p$ level from the ground $2 s$ level by H atoms. Allen \& Dickinson's estimates are substantially smaller than expected from Drawin's formula. The quantum mechanical ( $\equiv$ true?) rate is $100-200$ times smaller at the temperatures of interest $T \sim 2000-6000 \mathrm{~K}$ than the rates adopted by Steenbock and Holweger (1984). If the lower rate for $2 s \rightarrow 2 p$ excitation proves typical of rates for other transitions, Steenbock \& Holweger's calculations show that $\mathrm{H}$-atom collisions are not competitive with the electron-atom collisions in determining the departures from LTE in the excitation and ionization equilibrium of atoms.

Although it seems clear that additional quantum mechanical calculations are feasible for at least simple atoms, experimental confirmation of these results and particularly measurements on transitions not so readily amenable to theoretical attack would be of interest. One key problem is the production of a low-energy beam of $\mathrm{H}$ atoms. Since $k T \sim 0.2-0.6 \mathrm{eV}$ for cool stellar atmospheres, the rate constants under stellar conditions are usually dominated by the cross-section within $1 \mathrm{eV}$ of the threshold for excitation or ionization. Unfortunately, "no reliable hydrogen atom beam source for the 1-10 eV range is known" (Fleck et al. 1991). For energies above about $10 \mathrm{eV}$, photodetachment of an $\mathrm{H}^{-}$beam provides a useful $\mathrm{H}$-atom beam. Such a source was used to measure the emission cross-section for the $\mathrm{Na}$ I $3 p \rightarrow 3 s$ transition for $\mathrm{Na}$ colliding with $\mathrm{H}$ atoms of energies from 15-1000 eV. The results shown in Figure 7 are not exactly equivalent to the $3 s \rightarrow 3 p$ excitation cross-section because the collisions with $\mathrm{H}$ atoms can lead to population of higher $\mathrm{Na}$ I levels that populate the $3 p$ level through cascades. Certainly, the emission cross-section is an upper limit to the desired excitation cross-section for $3 s \rightarrow 3 p$.

The cross-section estimated from Drawin's expression (see Lambert 1993) is shown in Fig. 7. In the energy range of overlap with experiment, the Drawinian 


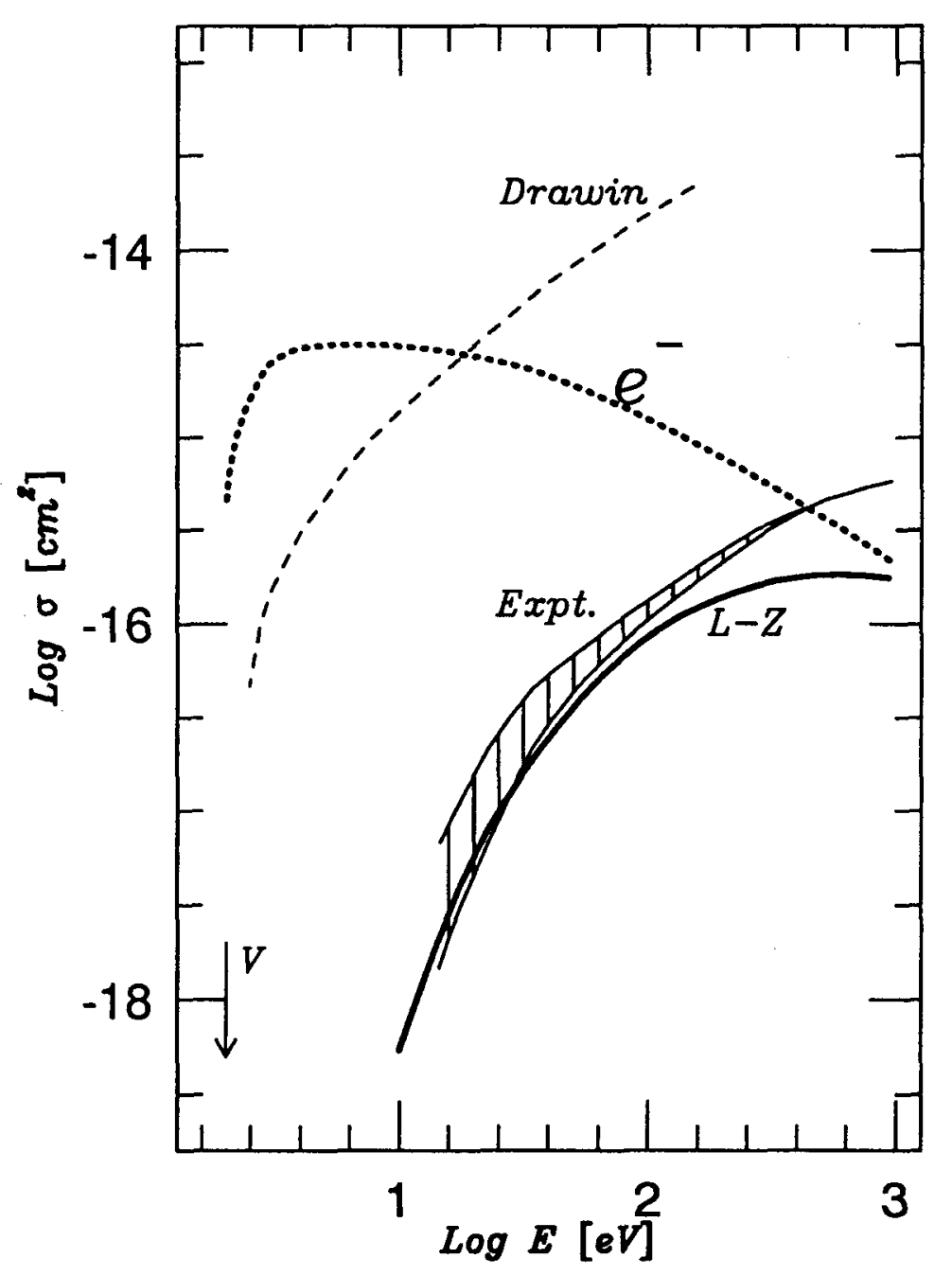

Fig. 7. Collision cross-sections for excitation $\mathrm{Na}$ I $3 s^{2} S \rightarrow 3 p^{2} P$ as a function of the laboratory energy of the projectile $\left(\mathrm{H}\right.$ or $\left.\mathrm{e}^{-}\right)$. Experimental estimates (upper and lower error bounds) for excitation by $\mathrm{H}$ atoms are shown for $E_{\mathrm{H}} \sim$ $10-1000 \mathrm{eV}$ by the hatched area (Fleck et al. 1991). Theoretical estimates for excitation by $\mathrm{H}$ atoms include the Landau-Zener model (see line marked L-Z), and the Drawinian prediction (Lambert 1993). The experimental cross-section for electron excitation is shown by the curve labelled $\mathrm{e}^{-}$. 
estimates are a factor of about 100 larger than the experimental results. The cross-section for electron excitation is also shown in Fig. 7. Near threshold, it is apparent that the cross-section of $\mathrm{H}$-atom excitation is likely to be 4 or more orders of magnitude smaller than that of electron excitation. In view of the lower thermal velocities of the $H$ atoms, it appears that the electrons will be greatly favoured in a comparison of the overall rates of excitation, i.e., $n_{e}\langle\sigma v\rangle_{e}$ vs. $n_{\mathrm{H}}\langle\sigma v\rangle_{\mathrm{H}}$.

A realistic calculation of the cross-section requires access to accurate potential energy curves for the $\mathrm{NaH}$ molecule. Fleck et al. (1991) examined computed ${ }^{1} \sum^{+}$potential curves. The $\mathrm{H}^{-}+\mathrm{Na}^{+}$ion pair potential introduces avoided crossings of potential energy curves. A collision pair can make a transition between potential energy curves at an avoided crossing. Fleck et al. (1991) use the Landau-Zener (LZ) model to estimate the $3 s-3 p$ emission cross-section and obtain a fair fit to the experimental results. Extrapolation of the LZ predictions to near threshold give $\sigma \sim 10^{-21} \mathrm{~cm}^{2}$. Fleck et al. (1991) report that they also undertook quantum mechanical scattering calculations which gave a much larger cross-section near threshold ("The origin of this discrepancy is not yet understood."). Since the rate constants at the low temperatures of the photospheres where $\mathbf{H}$-atom collisions are of potential importance are determined by the near-threshold cross-sections, it is critical that the theory be accurate in this region. Fleck et al. (1991) conclude that "the curve crossing mechanism is the essential mechanism" and "reliable quantum mechanical scattering calculations are required for the energy region between the threshold and the range of the present experimental data." This combination of conclusions make it unlikely that a general recipe can be found for the $\mathrm{H}$-atom excitation (and ionization) cross-sections. It is to be hoped that one or two atoms of astrophysical interest can now be examined in detail and the rate constants for $\mathrm{H}$-atom collisions applied to a non-LTE study.

The Massey criterion suggests that atoms seem likely to be effective in causing excitation between rotational and vibrational levels of electronic states of molecules. $\mathrm{H}, \mathrm{H}_{2}$, and $\mathrm{He}$ are the prospective contenders in the competition with electrons. There is laboratory data on $\mathrm{H}_{2}$ and $\mathrm{He}$ and a very limited amount on $\mathrm{H}$ as the cause of inelastic collisions with molecules. But much of this data involves molecules of little or no interest to the stellar spectroscopist.

\section{Information, Languages, and Communication}

In this review, I have attempted to set out why a particular group of cool starsthe AGB stars - are today of burning interest to observers and theoreticians in the astronomical village on the south bank of the river. I hope my fellow villagers will excuse the omissions and generalities that I was forced to make. They exist for several reasons but primarily because I was trying to beguile the villagers on the north bank into working with us in solving the fundamental questions presented by the stars: How and why do they evolve? and how are the chemical elements synthesized? 
By sketching a few topics in the spectroscopy of AGB stars, I trust that I have shown how "information" drawn from the northern village is needed by those in the southern village who wish to extract results ("information") about stellar evolution and nucleosynthesis from stellar spectra. There is a corresponding flow in the opposite direction; for example, the laboratory molecular spectroscopist may find previously unreported transitions in spectra of cool stars.

The reasons for and benefits of exchange of information over the bridge are, I think, abundantly clear. In an era of instant communication, it would seem churlish to complain that not all is well. I shall complain! In order for the communication between the villages to be optimally effective, we and you should speak the same language. Often, we do not. Therefore, I will end this review by urging that we should train our students to become acquainted with the topics studied across the river. Astronomy students (and their professors) should welcome exposure to the state of the current art of quantum chemistry and to the beautiful variety of new techniques for experimental spectroscopy. I hope that we may meet on another occasion to enjoy "En udsigt fra Broen."

\section{References}

Anderson, N. 1981, Comments At. Mol. Phys., 10, 133

Bauschlicher C.W. Jr., Langhoff S.R., Taylor P.R., 1988, Astrophys. J., 332, 531

Beer H., Macklin R.L., 1989, Astrophys. J., 339, 962

Blöcker T., Schönberner D., 1991, Astron. Astrophys., 244, L43

Boothroyd A.I., Sackmann I.-J., 1988 Astrophys. J., 328, 653 and 671

Burbidge G.R., Burbidge E.M., Fowler W.A., Hoyle F., 1957, Rev. Mod. Phys., 29, 54

Busso M., Gallino R., Lambert D.L., Raiteri C.M., Smith V.V., 1992, Astrophys. J., 399, 218

Cameron A.G.W., Fowler W.A., 1971, Astrophys. J., 164, 111

Chase M.W., Davies C.A., Downey J.R., Frurip D.J., McDonald R.A., Syverud A.N. (eds.), 1984, JANAF Thermochemical Tables, 3rd edition

Costes M., Naulin C., Dorthe G., 1990, Astron. Astrophys., 232, 270

Drawin H.W., 1968, Z. Phys., 211404

Drawin H.W., 1969, Z. Phys., 225483

Fleck I., Grosser J., Schecke A., Steen W., Voigt H., 1991, J. Phys. B, 24, 4017

Hinkle K.H., Lambert D.L., 1975, Monthly Notices Roy. Astron. Soc., 170, 447

Hinkle K.H., Lambert D.L., Wing R.F., 1989, Monthly Notices Roy. Astron. Soc., 238, 1365

Hollowell D., Iben I. Jr., 1989, Astrophy. J. (Letters), 340, 966

Huang Y., Barts S.A., Halpern J.B., 1992, J. Phys. Chem., 96, 425

Iben 1. Jr., 1975, Astrophys. J., 196, 525

Iben I. Jr., 1975, Astrophys. J., 196, 549

Iben I. Jr., 1991, Astrophys. J. Suppl., 76, 55

Jonnson J., Launila O., Lindgren B., 1992, Monthly Notices Roy. Astron. Soc., 258, $49 \mathrm{p}$

Käppeler F., Beer H., Wisshak K., 1989, Repts. Prog. Phys., 52, 945

Kjærgaard P., Gustafsson B., Walker G.A.H., Hultquist L., 1982, Astron. Astrophys., 115,145 
Lambert D.L., 1988, in The Impact of Very High $S / N$ Spectroscopy on Stellar Physics, ed. G. Cayrel de Strobel \& M. Spite, p. 563

Lambert D.L., 1991, in Evolution of Stars-the Photospheric Abundance Connection, ed. G. Michaud \& A.V. Tutukov, p. 299

Lambert D.L., 1993, Phys. Scripta, T47, 186

Lambert D.L., Clegg R.E.S., 1980, Monthly Notices Roy. Astron. Soc., 191, 367

Lambert D.L., Ries L.M., 1981, Astrophys. J., 248 , 228

Lambert D.L., Smith V.V., 1993, in preparation

Langhoff S.R., Bauschlicher C.W. Jr., 1988, J. Phys. Chem., 89, 2160

Larsson M., Siegbahn P.E.M., Ågren H., 1983, Astrophys. J., 272, 369

Lu R., Huang Y., Halpern J.B., 1992, Astrophys. J., 395, 710

Malaney R.A., 1987, Astrophys. J., 321, 832

Malaney R.A., Lambert D.L., 1988, Monthly Notices Roy. Astron. Soc., 235, 695

Massey H.S.W., 1949, Repts. Prog. Phys., 12, 248

Merrill P.M., 1952, Astrophys. J., 116, 21

Plez B., Smith V.V., Lambert D.L., 1993, Astrophys. J. in press

Sackmann I.-J., Boothroyd A.I., 1992, Astrophys. J., 392, L71

Scalo J.M., 1978, Astrophys. J., 221, 627

Scalo J.M., Despain K.H., Ulrich R.K., 1975, Astrophys. J., 196, 805

Schwarzschild M., Härm R., 1967, Astrophys. J., 246, 251

Seaton M.J., 1962, in Atomic and Molecular Processes, ed. D.R. Bates, 1962, Chapter 11

Sauval A.J., Blomme R., Grevesse N. 1994, in Poster Session Proceedings of IAU Coll. 146, eds. P. Thejll \& U.G. Jørgensen, p. 107

Smith V.V., 1988, in The Origin and Distribution of the Elements, ed. G. J. Mathews, p. 535

Smith V.V., Lambert D.L., 1984, Publ. Astron. Soc. Pacific, 96, 226

Smith V.V., Lambert D.L., 1990a, Astrophys. J. (Letters), 36, L69

Smith V.V., Lambert D.L., 1990b, Astrophys. J. Suppl., 72, 387

Smith V.V., Lubowich D., Plez B., Lambert D.L., 1993, in preparation

Thompson R.I., 1973, A strophys. J., 181, 1039

Thomson J.J., 1912, Phil. Mag., 23, 449

Tomkin J., Lambert D.L., 1983, Astrophys. J., 273, 722

Truran J., Iben I. Jr., 1977, Astrophys. J., 216, 797

Tsuji T., 1988, Astron. Astrophys., 197, 185

Ulrich R.K., 1973, in Explosive Nucleosynthesis, eds. D.N.Schramm\&D.W.Arnett, p.139

Uus U., 1973, Soviet Ast., 17, 196 